Winfried Wehle

(Eichstätt)

\title{
DAS ANDERE IM EIGENEN. ZUR PASTORALEN EROBERUNG DER ANTHROPOLOGIE IN DER (ITAL.) RENAISSANCE
}

Die Entdeckung und Eroberung Spanischamerikas war nicht nur ein politisches, wirtschaftliches und weltanschauliches Ereignis. Sie gab gleichermaBen zu einer anthropologischen Herausforderung ersten Ranges Anlass. Man könnte geradezu von einer Hermeneutik der Entdeckung sprechen. Sie mutete, vor allem in der ersten Generation, dem mitgebrachten Menschenbild eine unerhörte Prüfung seiner Grundlagen zu. Wenn sich die Eroberer sin der Neuen Welt nicht fremd fühlten`, dann zunächst, weil sie die >Anderen als fremd empfanden': Sie lösten ihr Problem der Alterität durch die Verschärfung ihrer eigenen Identität. Ihre Weltreligion hatte das Neue immer schon vorgesehen als den noch ausstehenden Teil eines Ganzen, das sich christlich erfüllen sollte. Insofern praktizieren sie eine >Selbstdurchsetzung «, die - nach der mächtigen Endzeittheologie des kalabrischen Abtes Joachim da Fiore - das eigentliche Anliegen des Hl. Geistes war. Diese Hermeneutik der Erfüllung leitete noch Kolumbus an. ${ }^{2}$ Er hatte damit seiner Entdeckung zugleich ein exemplarisches Deutungsmuster an die Seite gestellt. Dass sich `Erfüllung« von Vereinnahmung nur schwer unterscheiden lässt, tat gewiss ein Übriges, um das Fremde und Fernstehende als Unerlöstes anzusehen und es sich ritterlich-heroisch anzueignen. Dadurch würde das messianische Werk der Vollendung der Welt unerhört beschleunigt. Wer die Welt solchermaßen totalisieren will und darf - zumal christlich -, kann er der Versuchung widerstehen, dabei nicht totalitär zu werden?

Es wäre - theoretisch, im Sinne einer alternativen Hermeneutik - möglich gewesen. Denn während die Aneignung Americas die Vorstellung von

1 Wie Dieter Janik zu bedenken gibt. Cf. id. / Wolf Lustig (1989: 14). Das gleiche Problem hat er unter dem Aspekt des mestizaje eingehend behandelt, cf. Dieter Janik (1994).

2 Cf. Winfried Wehle (1995). 
einem Hirten, einer Herde, einem Diskurs über Welt und Mensch forcierte, fand gleichzeitig eine ganz andere Entdeckung statt. Über ihr waltete nicht die Aura des Weltgeschichtlichen. Auch war sie nicht eigentlich eine terra incognita, vielmehr versunkenes Kulturgut. Ihre Wiederaneignung wurde deshalb auch renovatio, restitutio, reformatio - als kulturelle Wiedergeburt bezeichnet. So suchten Humanismus und Renaissance in Italien sich der conditio humana erneut zu vergewissern. Gleichwohl sprach auch sie, auf ihre Weise, dem Menschen ein neues Selbstverständnis, dignitas hominis, zu. Und dies war wahrlich ein >Anderes` gegenüber der mittelalterlichen miseria hominis. Doch wo auf der einen Seite mit Waffen, Technik, Strategie menschliche Selbstermächtigung unter Beweis gestellt wurde, ließ sich auf der anderen gerade behaupten: Wenn der Mensch wahrhaft sich selbst verwirklichen will, dann hat er sich mit den Waffen der Sprache zu erobern. So stehen sich zwei höchst gegensätzliche Modelle der Selbstverwirklichung gegenüber: ein sendungsbewusstes >Macht euch die Erde untertan dort, hier aber das kommunikative Gebot: , Geht sprachlich aufeinander ein . Das eine hätte dem anderen durchaus als - humanes - Korrektiv entgegenkommen können. Denn beide Entdeckungen fanden nahezu gleichzeitig statt und doch haben sie sich - vielleicht gerade deshalb - auf historische Weise verfehlt. Wer weiß, wie die Entdeckten behandelt worden wären, wenn die Entdecker sie mit dem Menschenbild hätten betrachten können, das neben ihnen gerade entworfen wurde.

In dessen Mittelpunkt steht die bis heute bewegende Frage, wie weit menschliche Identität ohne Sprache - im weitesten Sinne - überhaupt denkbar ist. Wer dies, wie im Renaissancehumanismus, mit Entschiedenheit verneint, der, so die historische Konsequenz, findet in der Kunst der Sprache die Führung in Menschendingen.

Pico della Mirandola hat sich dazu beispielhaft in einem »der edelsten Vermächtnisse der Renaissance geäußert $\ll^{3}$, in seiner Oratio de hominis dignitate aus dem Jahre 1486. ${ }^{4}$ Ihre berühmteste Stelle leitet aus dem biblischen Schöpfungsbericht die Genesis einer anthropologischen Welt ab. Gottvater, der höchste Künstler, setzt Adam, sein Geschöpf, so in sein Eigenrecht ein:

3 Jacob Burckhardt (1952: 330).

4 Giovanni Pico della Mirandola (1990). 
Wir haben dir keinen festen Wohnsitz gegeben, Adam, kein eigenes Aussehen noch irgendeine besondere Gabe, damit du den Wohnsitz, das Aussehen und die Gaben, die du selbst dir ausersiehst, entsprechend deinem Wunsch und Entschluß habest und besitzest. Die Natur der übrigen Geschöpfe ist fest bestimmt und wird innerhalb von uns vorgeschriebener Gesetze begrenzt. Du sollst dir deine ohne jede Einschränkung und Enge, nach deinem Ermessen, dem ich dich anvertraut habe, selber bestimmen. Ich habe dich in die Mitte der Welt gestellt, damit du dich von dort aus bequemer umsehen kannst, was es auf der Welt gibt. Weder haben wir dich himmlisch noch irdisch, weder sterblich noch unsterblich geschaffen, damit du wie dein eigener, in Ehre frei entscheidender, schöpferischer Bildhauer dich selbst zu der Gestalt ausformst, die du bevorzugst. Du kannst zum Niedrigeren, zum Tierischen entarten; du kannst aber auch zum Höheren, zum Göttlichen wiedergeboren werden, wenn deine Seele es beschließt. ${ }^{5}$

Wert und Würde des Menschen sind aus seiner Stellung in der Schöpfung abgeleitet. Sie bleibt das Maß aller Dinge. Pico bemüht - sei es gläubig, sei es strategisch - die theologische Seinshierarchie: Dass alles seinen festen Rang auf der Himmelsleiter der Schöpfung habe. Doch er identifiziert den neuen Adam gerade als denjenigen, der sich ihrer Systematik nicht fügt. Inmitten einer durchgängig bestimmten Welt ist er das einzig Unbestimmte, ein Fremdling des Systems. Positiv gewendet: Ihm allein ist, im Rahmen des Geschaffenen, die Freiheit der Selbstbestimmung eingeräumt. ${ }^{6}$ Wird er darin aber nicht bereits als ein Spielraum begriffen, der ihm eine eigene Gestaltungsmöglichkeit eröffnet? Der ihn Mensch nach seinem - freien Willen und seiner eigenen Vorstellung sein lässt? Er träte damit von Ferne bereits ins Konzept von Subjektivität ein. Vormodern wäre es in dem Sinne zu nennen, dass es nicht um den Einzelnen als solchen geht, sondern um die Gattung Mensch in ihrer Andersartigkeit innerhalb des großen Zusammenhangs der natura naturata. Pico fasst sie schon als offenen Kontext im Rahmen fester, göttlich »vorgeschriebener Gesetze« auf: begrenzt von einer Ewigkeit, aus der er entlassen, und einer, in die er, erlöst oder nicht, zurückkehrt. Zwischen beiden aber tut sich die Erfahrung der Zeit auf. Sie eröffnet gleichsam einen dritten, beweglichen Ort zwischen dem unwider-

5 Giovanni Pico della Mirandola (1990: 6-7). Zur Verbindung mit Ficinos Neuplatonismus cf. Ernst Cassirer ( ${ }^{5} 1977$ : 139ss.), der »die Liebe als einen wechselseitigen Prozeß« fasst und sie damit aus dem platonischen Aufstiegsschema heraustreten lässt. Der Begriff des Menschen bei Pico entspricht genau der Liebestheorie Ficinos.

6 Wenn er auch, mit Ficinos Platonismus, als Verwirklichungsziel des Menschen seine geistige Identität festhält. Aber es handelt sich um die »erhabene und heilige Philosophie« der Naturerkenntnis; und ihr Prinzip ist weniger die Stufenleiter der Theologie, sondern die Einsicht, ja der Eintritt in die sympatheia, die innere Wechselbeziehung des Universums, cf. Giovanni Pico della Mirandola (1990: 56ss.). 
ruflichen Anfang und Ende - eine temporale Identität, die den Durchgang von der Geburt zum Tod als einen eigenen, begrenzten Zeit-Raum wahrzunehmen vermag, als Frist eines irdischen Lebens. Eine solche Existenz um ihrer selbst willen wollte allerdings gelernt sein. Dem dienten nicht nur die pragmatischen artes vivendi. Picos neuzeitliches Manifest zeichnet sich gerade dadurch aus, dass er diesem Leben in der Zeit auch eine brisante Handlungstheorie gibt. Denn die Autonomie, die er dem Menschen gewährt, erwächst ihm aus einer Analogie zum Schöpfer aller Dinge. Dem Menschen im Kleinen zu erlauben, was Gott im Großen tut - liegt dem nicht, strenggenommen, eine kühne Einschränkung, ja Leugnung, anders gesagt, Usurpation seiner Schöpfungsmacht zugrunde? Gewiss, Pico sprach, in guter theologischer Tradition, vom Menschen als einer Gattung innerhalb des Geschaffenen. Dennoch verdankt sich seine Andersartigkeit einer Seinsdifferenz, also dem, worin er gerade aus der sonst überall waltenden kosmologischen Gesetzesmäßigkeit herausfällt. Er kann - oder muss dadurch - sein eigenes Leben leben.

Die Folgen dieser programmatischen Freisetzung des Menschen zu sich selbst waren beträchtlich. Die wohl folgenreichste hat schon Pico selbst entfaltet. Als Mitte der Welt, frei nach eigenem Ermessen sich entfalten zu können: Für diese Ausnahmestellung des Menschen gibt es nur einen legitimen Archetypus, eben den Welterschaffer selbst. Doch stellt Pico damit im Grunde nicht insgeheim die theologische Schrifthermeneutik auf den Kopf? ${ }^{7}$ Statt aufsteigend, im Sinne der analogia entis, von den mangelhaften Geschöpfen auf den vollkommenen Schöpfer zu schließen, hat er, von ihm ausgehend, ein schöpferisches Wesen auch des Menschen abgeleitet. Der Mensch - ein kleiner Gott; Mikrokosmos im Makrokosmos. Seine Sündhaftigkeit wäre zurückgenommen und machte einer neuen Weltwürde Platz, die ihm den Glanz einer Wiedergeburt verleiht.

Sie hat jedoch ihren nicht minder neuzeitlichen Preis. Denn was er aus seiner schöpferischen Lizenz macht, hängt jetzt in hohem Maße von ihm selbst ab. Deshalb sieht er sich, gewissermaßen als Folgelast seiner Selbstbestimmung, vor die Aufgabe der Selbsterkenntnis und -verantwortung gestellt. Um zu erfahren, was er kann, muss er wissen, wer er ist. Folgerichtig

7 Eine der grundsätzlichen Ablösungen der Neuzeit vom Mittelalter, die Reinhold R. Grimm (1981: 567-576) als Übergang »von der explikativen zur poetischen Allegorie« gewürdigt hat und darin zugleich die entscheidene Neuerung gerade auch der Arcadia Sannazaros sieht. 
gipfelt Picos Entwurf im delphischen `Erkenne dich selbst.. »Wer sich nämlich erkennt«, fügt er hinzu, »erkennt in sich alles. « ${ }^{8}$ Es ist dies der subjektivistische Grund seines neuen Lebens, insofern er sich dadurch als Objekt seiner selbst setzt. Ihm verdankt sich auch die Entstehung einer ganz neuen Wissenschaft von Menschen, die Anthropologie. Ihr Name wurde von Magnus Hundt mit dem bezeichnenden Titel eingeführt: Anthropologium de homine dignitate(!) natura et proprietatibus (1501). Eine hochinteressante Schrift. Durchaus der absteigenden Hermeneutik Picos vergleichbar, trennt er aus der Theologie eine Lehre des menschlichen Körpers heraus, die Anatomie. Sie antwortet auf die unterstellte Frage: Was ist der Mensch, wenn er nicht mehr allein auf Gott hin entworfen wird? Er wird darüber zum Schöpfer der Wissenschaft, die zwar vom metaphysischen Urheber aller Dinge ausgeht, das Geschöpf >Mensch` aber vornehmlich >physisch <, das heißt für sich betrachtet. Und: Er wird zum Künstler. Pico selbst deutet diese Konsequenz schon an. Der Mensch als »frei entscheidender, schöpferischer Bildhauer « - hat er sich von Gott nicht insgeheim einen Begriff nach seinem Bild und Gleichnis gemacht, auch wenn er ihn als höchsten Künstler respektiert? Außerdem: Die Schöpfung als Kunstwerk zu preisen, hieße dies dann nicht, dass menschliche Kunstwerke nicht länger als `Lügen verurteilt werden müssen, weil es nur die eine, offenbarte Wahrheit gibt? Wäre nicht vielmehr Kunsthandeln geradezu tätiger Gottesdienst, insofern der Mensch dabei besser als irgendwo anders »ohne jede Einschränkung und Enge« sich »selbst zu der Gestalt ausformen« kann, »die er bevorzugt«? In der Kunst wäre er demnach seinem Schöpfer am nächsten.

Eine curiositas kam dadurch über die Welt und ihn selbst. Wem das Recht eingeräumt wird, um sie und sich frei nach »eigenem Ermessen selber zu bestimmen «, muss die Bedingungen seiner selbst ungleich besser kennen als wenn ihm ein Lebensplan orthodox vorgeschrieben wird. Welches aber wären die Voraussetzungen, die einer solchen produktionsästhetischen Anthropologie gutzuschreiben wären? Pico geht abermals von der offiziellen Menschenlehre aus. Adam kann, sagt er, zum Niedrigen, Tierischen »degenerieren« oder aber zum Höheren, Göttlichen »regenerieren«. Er sieht sich damit einem heftigem Seelenkampf ausgesetzt. Um es mit aufklärerischer Begrifflichkeit zu sagen: Der Mensch ist das Resultat einer

8 Giovanni Pico della Mirandola (1990: 23-24). 
Psychomachia, in der Sinnlichkeit und Verstand um seine Identität kämpfen. Pico spielt dabei unverkennbar auf Marsilio Ficinos platonische Psychologie und, über ihn zurück, auf Thomas von Aquin und seine maßgebliche Bestimmung des Menschen als einer Doppelnatur, als einem animal rationale an. ${ }^{9}$ Sie fassen ihn also als eine coincidentia oppositorum elementarer Grundbestrebungen auf. ${ }^{10}$ Innerhalb der niederen, geistlosen Natur nimmt er, als Geistbegabter, die lichteste Stelle auf der dunklen Seite des Kosmos ein. Von Gott, der Allvernunft her gesehen, steht er aufgrund seiner animalischen Teilhabe jedoch auf der niedrigsten Stufe »himmlischer Lebewesen«. Pico hat ihn deshalb eigentlich bereits als ein problematisches Wesen situiert. Es kann sich frei selbst bestimmen, gewiss, aber für lange Zeit nur zwischen zwei unhintergehbaren Bestimmtheiten. Deutlicher gesagt: Es darf die Mitte seiner eigenen Welt bilden, aber diese Mitte ist, in letzter Konsequenz, leer und es sich selbst ein Fremder. Deshalb wird ihm negative Subjektivität abgenötigt. Was immer es auch aus sich machen mag - mehr als am Ergebnis bemisst sich seine Humanität an seiner vorrangigen Aufgabe: Um Mitte sein zu können, muss es zuerst die widerstrebenden Anlagen des Leiblichen und Seelischen in sich ver-mitteln. Ein unerhörter Gedanke. Denn die ihm aufgetragene Selbstvereinbarung ist, im Prinzip schon hier, ein offenes Projekt. Picos Schrift wurde denn auch sogleich verboten; er selbst eingesperrt. Dabei hatte er sich mit allen verfügbaren Lehrmeinungen abgesichert und als Vermittlungsziel die geltende, das heißt christliche Moral eingesetzt. Und natürlich sollte der neue Adam sich, das war humanistisches und christliches Gemeingut, nach oben, zum Vernünftigen, Geistigen hin entwickeln.

Wie aber, und daran vor allem hatte sich die Würde des Menschen zu bewähren, lässt sich diese schöpferische Verwiesenheit auf sich selbst durchführen? Mit anderen Worten: Sie bedarf Formen des Umgangs mit sich selbst - Anthropologie. Nur mit einem eigenen Logos ist ein selbstbestimmter Mensch zu machen. Pico geht darauf lediglich am Rande und unsystematisch ein. Aber zumindest umrisshaft ahnt schon er die drei Weisen der Selbstfindung, die dem neuen Menschenbild eine lange Zukunft sichern werden. Am offensichtlichsten ist die konventionellste: Christliche wie antike Lebenslehre waren sich einig, dem zwischen Gut und Böse hin- und

9 Cf. die historische Übersicht von Michael Landmann (Hrsg.) (1962: 112-160), hier pp. 128ss.

10 Weniger im Sinne des Cusaners als von Giordano Bruno. Cf. Nuccio Ordine (1999). 
hergerissenen Menschen mit Hilfe der Rhetorik im besten, mit Strafen im anderen Falle Mores zu lehren. Das wird so bleiben bis zu Kants moralischem Imperativ. Daneben leitet Pico jedoch aus der menschlichen Teilhabe an Himmel und Erde eine magische Naturphilosophie ab, die zur Grundlage einer Wissenschaft von der Natur wird. Und, drittens, der Mensch wird Mensch durch Kunst. Stets aber, und dies war vielleicht die bedeutendste humanistische Vorleistung, verband sich der Anspruch auf eine dignitas hominis mit der Auffassung, dass die Aneignung eines Selbst sich allererst der Beherrschung der Sprache verdankt. ${ }^{11}$ Mit einer ars bene dicendi vor allen Dingen lässt sich das Niveau >Mensch` gegenüber den Befremdungen des Animalischen (und eines strafenden und rächenden Gottes) halten. Sie vor allem macht, in humaner Perspektive, den Menschen zum Menschen. ${ }^{12}$ Mit Berufung darauf und auf Orpheus, Zarathustra, die Kabbala und anderes hatte der Philosoph eine eigene theologia poetica im Sinn, mit der er begründen wollte, dass selbst aus der »Dunkelheit der Geschichten « eine ganz »urtümliche Weisheit« spricht. ${ }^{13}$ Sie wurde nie geschrieben. Pico starb, im Banne Savonarolas, mit zweiunddreißig Jahren.

II.

Doch was ihm versagt war, haben die Künste ihrerseits zu klären versucht. Früher als die Philosophie haben vor allem sie sich einer Renaissance verschrieben und sie auf vielerlei Feldern der Darstellung vollzogen. Eine fand die besondere Vorliebe von Künstlern und Publikum: es war Arkadien, die Welt pastoraler Literatur, Malerei und Musik. Heute ist sie entschwunden, wie andere irdische Paradiese. Damals jedoch und für mehr als zwei Jahrhunderte war sie eines der meistbesuchten imaginären Reiseziele. Dieser überwältigende Erfolg ist erstaunlich. Er steht, sieht man von spektakulären Aufmachungen wie dem Pastor Fido von Guarini ab, in einem auffälligen Gegensatz zur ereignisarmen, beschaulichen, weltfernen Art von Land und Leuten. Sie müssen offensichtlich im kultivierten Publikum ein vitales In-

11 Seit den Anfängen des Humanismus, namentlich durch Ciceros Schriften, eine Grundüberzeugung. Cf. Winfried Wehle (1993).

12 Dazu Jürgen Trabant (1997: 595ss.).

13 Giovanni Pico della Mirandola (1990: 57-58), wo es heißt, die Magie des dichterischen Wortes sei es, welche sdie in den Tiefen der Welt, im Schoß der Natur, in den geheimen Speichern Gottes verborgenen Wunder ans Licht bringt《. 
teresse getroffen haben, das ihr Bild höchst ansprechend fand. Vieles deutet darauf hin, dass hier mittels der Kunst die - anthropologische - Grundfrage verhandelt wurde, wie der Mensch mittels Kunst Mensch werden kann. Und dass es hinter der Harmlosigkeit des Landes tatsächlich um Brisantes gegangen sein muss, zeigt seine Geschichte. Je mehr es sich neben epischen, tragischen und komischen Fällen menschlicher Verstrickung behauptete, desto größer war offenbar die Notwendigkeit, die naturverbundene Sittlichkeit der Schäfer gegen verhärtete Ehrbegriffe außerhalb ins Feld zu führen. Die Kontroverse ist historisch geworden in Torquato Tassos befreiendem »Erlaubt ist, was gefällt« (Aminta I, 2), dem Battista Guarini mit dem angepassten »Erlaubt ist, was sich ziemt« (Pastor Fido IV, 9) sogleich ins Wort fiel. In Arkadien ging es, zumindest zu Zeiten der Renaissance, um einen neuen Begriff vom Menschen.

Das Land war wie geschaffen dafür. Es liegt prinzipiell draußen vor jeder Zivilisation. Es huldigt einem Ideal des einfachen Lebens, das weitgehend von allem negotium suspendiert. Schäfer und Nymphen, seine Bewohner, führen dadurch eine Art Sonntagsleben. Diese Entlastung von materiellen und sozialen Zwängen verschafft ihnen das nötige otium, um sich vor allem mit sich selbst beschäftigen zu können. Die arkadische Ansicht der Welt nimmt gewissermaßen eine "phänomenologische Reduktion« (Edmund Husserl) vor der Zeit vor: Die äußere Tatarmut der Leute dient der Entfaltung ihres Innenlebens und Gefühlsreichtums. Angestoßen wird alles durch eine der ältesten Dramaturgien der Kunst, die Liebe. Schäfer sind - in aller Regel unglücklich - verliebt. Arkadien tritt dabei zwar materiell das Erbe der petrarkistischen und antiken Liebesdichtung, nicht jedoch deren privative Lösung an: Hier soll Liebe um ihrer Erfüllung willen besprochen werden. Was den Menschen von Natur aus elementar bewegt, kommt damit als Teil, nicht mehr nur als - sündhaftes - Gegenteil seiner Menschwerdung in Betracht.

Die Autoren, die das literarische Bild Arkadiens prägten, Sannazaro, Montemayor, Tasso, Guarini, Honoré d'Urfé, Marino haben in diesem Land eine bedeutsame semiotische Entdeckung gemacht. Das bukolische und liebeslyrische Inventar ließ sich umfassend metonymisch in Anspruch nehmen. Dadurch entstand die arkadische Blickerweiterung schlechthin: Sie nahm im Verhältnis von liebendem Schäfer und abweisender Nymphe die menschliche Doppelnatur des animal rationale wahr. In der Nymphe, dem weiblichen Part, war das animale, die naturhaft kreatürliche Teilhabe 
des Menschen eingetragen. Die Dienerin der Diana weiß kaum etwas von sich; entsprechend unbedingt gehorcht sie deshalb ihrem Versprechen, das sie der Göttin gegeben hat. In deren Namen jagt und tötet sie das Wild. Es ist ihr Erkennungszeichen: Ihre eigene Natur ist `wild`, weil fremdbestimmt, unerlöst und damit diskret Ausdruck für die Gefährlichkeit der unbewussten Natur. Sie kennt sich selbst nur als Wesen einer anderen Autorität; spricht wenig oder gar nicht, zumindest anfänglich. Für den Schäfer hingegen wird das rationale verfochten. Er hütet die Tiere und schont ihr Leben, das heißt er kultiviert die Natur und vertritt damit ihre entgegenkommende Seite. Die Nymphe wendet ihre Lebensenergie nach außen in die Verfolgung und Vernichtung des Animalischen; von daher ihre rigorose Keuschheitsverpflichtung. Er hingegen dringt auf seine Gefühle ein, wendet sich also nach innen, auf sich selbst. Seine Energie geht nicht in Taten, sondern in die Sprache. Während sie praktiziert, theoretisiert er, was den Menschen von Natur aus bewegt. Anlass dazu gab die innere Verwundung, die sie, die Jägerin, ihm zugefügt hat, als er sie angeschaut hatte und ihn dadurch die Pfeile Amors trafen. Dass sie jedoch so viel Macht über ihn hat, zeigt, dass auch das rationale< Prinzip, unter dem er steht, im Grunde auf seine Art ebenfalls unerlöst ist. Auch der Schäfer ist also nicht Herr seiner selbst: Er braucht sie, sonst ist er von Sinnen, das heißt sich selbst entfremdet. Um dies zum Ausdruck zu bringen, macht er ein synästhetisches >Bild des Jammers: seine Klagelieder. Beide zusammen aber demonstrieren für die neue anthropologische Auffassung, dass die gedankliche und kreatürliche Seite des Menschen zusammengehören, trotz ihrer Gegensätze. Wo dies nicht so ist, wie zu Beginn der arkadischen Liebeshändel, erleidet jeder seine anthropologische Rolle als (melancholische) Vereinseitigung des Prinzips, das er verkörpert. Die Frau ist sich fremd, weil sie zuwenig auf sich selbst bezogen; er, weil er zuviel mit sich selbst beschäftigt ist.

Doch in Arkadien finden sie die denkbar besten Voraussetzungen für eine Heilung ihrer Dezentrierung. Denn Schönheit, Anmut und Geneigtheit von Land und Leuten zeigen, dass sie, im weiteren Sinne, dem Horizont einer letzthinigen Versöhntheit unterstehen, auf den ihre Welt ausgerichtet ist: das Goldene Zeitalter und das paradiesische Glück des ersten Menschengeschlechtes. ${ }^{14}$ Dieses war in höchstem - naivem - Einvernehmen mit sich, weil jeder nur das wollte, was die Natur ihm gab und die Natur ihm 
von sich aus bot, wonach er verlangte. Vor allem aber, weil Tiere, Menschen und Götter noch eine Sprache hatten - jene >orphische`Ursprache, an der sich kulturell erschöpfte Epochen regelmäßig zu erneuern hoffen. Gewiss, dieses Ideal war unwiederbringlich dahin; alle - Arkadier, Dichter und Publikum - wussten es. Aber es blieb eine mythische Himmelserinnerung und damit Berufungsinstanz für ein menschenmögliches Glück - auch wenn davon kein neues Paradies zu erwarten war.

Es ist nun überaus bemerkenswert, wie die kleine, pastorale Gemeinde versucht hat, die inneren (und äußeren) Gegensätze eines animal rationale auszutragen und zu besänftigen. Heroischer Tatendrang ist ihrem locus amoenus fremd. Umso mehr kam es auf seine alternativen Waffen der Selbstbehauptung an. In Arkadien wird die Sprache zum Ereignis. Sie steht, in allen ihren Spielarten, im Mittelpunkt dieser Sonderwelt. Wenn eine Aussicht auf Aussöhnung des menschlichen Dualismus besteht, dann, so lautet die These dieses Landes, mit Hilfe der Sprache. Das Glück, von dem hier überall die Rede ist, lässt sich nicht mit Waffen, sondern nur mit arkadischem Logos erlangen. Für die gebildeten unter seinen Liebhabern muss dies eine erregende Vorstellung auf den zweiten Blick gewesen sein. Seit Boccaccios Decameron hatte es wohl keinen anderen kulturellen Ort gegeben, der es erlaubt hätte, im Schutze von Fiktionalität den Wert der Sprache für einen humanen Begriff vom Menschen so hoch zu veranschlagen.

III.

Sannazaro hat das Verdienst, als erster aus der geläufigen bukolischen Materie eine arkadische Welt herausgehoben zu haben. Innerhalb weniger Jahrzehnte gehörte dieses Hirtenland dann zum festen Bestand imaginärer Ergänzungswelten.

Seine Arcadia mag deshalb einsichtig machen, worin die besondere Faszination dieser ästhetischen Projektion bestand. Zunächst nimmt sie durch ihre alles umfassende Stimmigkeit für sich ein. Pflanzen, Tiere und Menschen, der ganze untere Bereich des Kosmos, ist von der gleichen, einvernehmlichen Gesinnung durchdrungen. Bereits die rhetorische Aufzählung der Baumarten zu Beginn dient diesem Zweck: Sie führt das friedliche Miteinander der unterschiedlichsten Gewächse vor. In ihm wirkt sich aus, was die Natur allererst zu dieser Harmonie befähigt: dass sie, selbst wo sie für uns stumm bleibt, gleichwohl einen kommunikativen Zusammenhang 
bildet. Natur ist hier, ihrem Wesen nach, Sprache. Der Wald flüstert; die Quellen und Bäche murmeln; die Vögel singen; der Wind haucht. ${ }^{15}$ Jedes ihrer Elemente hat seine ursprüngliche Stimme. Das gilt artgemäß auch für die Natur des Menschen. Sein unmittelbarster Ausdruck ist, nach arkadischer Auffassung, Musik und Gesang. Diese alles durchwaltende Gestimmtheit hat in Pan ihr Sinnbild gefunden, dem Gott der Hirten, dessen Flöte Emblem für diese mythische Ursprachlichkeit der Natur ist. ${ }^{16}$ Auf sie berief sich die Sage vom Goldenen Zeitalter, als sie die Kindheit des Menschengeschlechts als eine Zeit grenzenloser Kommunikation beschwor. Die ganze Welt war damals noch im »Einklang «. ${ }^{17}$ Diesen emphatischen Zauber wertet Arkadien zu einer eigenen Sprache des animale auf. Sie zeichnet den Menschen ebenso aus wie seine >rationalen` Ausarbeitungen. Rückblickend auf die orphischen Mythen bzw. das christliche Pneuma hat auch Arkadien Anteil an einer Wendung in die Neuzeit, wo die erkenntnistheoretische Saat dieser Ursprache dann bei Rousseau, Vico, Hamann, Herder, Humboldt oder Novalis ganz aufgehen wird: in der Kritik aufklärerischer Vernunft.

Auf diese >magische< Natureinfalt ging das Glück der ersten Menschen zurück. Auf sie beziehen sich - noch immer - die Klagelieder der Schäfer: Wenn sie hier ihr Unglück anstimmen, dann kann es sich immerhin als Verlust artikulieren gegenüber einer uranfänglichen Versöhntheit der äußeren und inneren Natur. Geht nicht wenigstens noch das \Echo< sympathetisch auf ihre Verstimmung ein? Und zeugen nicht heute noch die turtelnden Tauben davon, wie es einst war? Ursache für den selbstverschuldeten Ausgang aus der Natürlichkeit aber ist, seit Vergil schon, dass der Mensch, einem alten Dekadenzschema zufolge, zivilisatorisch bis in ein Eisernes, ja Bleiernes Zeitalter - Blei für Kugeln - heruntergekommen und seinen Ursprüngen gänzlich entfremdet ist. ${ }^{18}$ Zwar kennt er noch natürliche Wünsche; sie natürlich befriedigen, wie im Goldenen Zeitalter, kann er allerdings nicht mehr. Sannazaro hat seine Schäfer deshalb zu Flüchtlingen ge-

15 Als literarischer Topos war dieser locus amoenus selbst dem Mittelalter nicht fremd geworden. Cf. Ernst R. Curtius (1948: 202 [Kap. $10 \S 6$ »Der Lustort«]). Erst Arkadien erfasst in der Eloquenz des Ortes ein Sprachereignis. Cf. Jacopo Sannazaro (1990: 14 [n. 20] und 165-170).

16 Vor ihm steht, maßgeblich durch Ficinos neuplatonische Akademie geprägt, Orpheus, der mythische Sänger als Sinnbild ursprünglicher Poesie. Cf. dazu Richard Cody (1969: 23ss.).

17 »[...] i tempi antichi, quando i buoi parlavano, / che 'I ciel più grazie allor solleva producere. / Allora i sommi dii non si sdegnavano / menar le pecorelle in selva a pascere; / e, come or noi facemo, essi cantavano«; in: Jacopo Sannazaro (1990: 113 [VI, vv. 69-73]).

18 Cf. Gustavo Costa (1972). 
macht, welche die Zivilisationskrankheiten von Hof und Stadt nach Arkadien tragen, um sie dort nach Art des Landes, das heißt sprachlich, mit arkadischer Logotherapie zu behandeln.

Für die Schäfer heißt dies Diskurswechsel: Sie hatten von der krankmachenden Kultursprache zu lassen, um an der Natursprache Arkadiens zu gesunden. Anders gesagt: Ihr verbildeter Verstand soll sich an den sinnlichen `Quellen< des Lebens erneuern. Im Grunde ein unerhörtes Programm. Es sieht in der niedrigen Natur nichts weniger als eine Gegenkultur, die als gleich- und eigenwertig ins Bild des animal rationale gehört. Sannazaro behauptet andererseits jedoch nur, was Botticellis anthropologisches Andachtsbild von der Geburt der Venus allegorisch erzählt. Venus verkörpert, im besten Sinne des Wortes, die schöne Möglichkeit des Menschen zwischen zwei gegenläufigen Ansprüchen. Auf der einen Seite die sinnliche, genetische Energie der Natur - das sanimalische` Moment der Windgötter. Auf der anderen kommt ihr, in Gestalt der Frühlingshore, die Repräsentantin der Künste entgegen, deren Tugenden - das >rationale< Moment - ihr einen reich verzierten Mantel rhetorischer Zucht überzulegen vermag. Venus selbst wird zwar lebhaft von beiden angemutet. Ihre Schönheit und Würde entspringt jedoch gerade daher, dass sie - nackt - weder das eine noch das andere, sondern das Ankommende, Neue, die Mitte zwischen beiden, ein Drittes, ihre Vermittlung ist, die den Geist natürlich und die Natur geistig macht. Ihre Nacktheit - ist sie so gesehen dann nicht Zeichen für ein kulturell ungebrochenes Idealbild des neuen Menschen, den Gott, um mit Pico zu sprechen, als »ein Geschöpf von unbestimmter Gestalt« schuf, dessen paradiesische Anfänglichkeit gerade die Voraussetzung dafür wäre, um sich neuzeitlich selbst zu erfahren: sich »ohne jede Einschränkung und Enge, nach seinem Ermessen [...] selber zu bestimmen«? Die Geburt der Venus: Allegorie vom Werden einer neuen, natürlichen Humanität ${ }^{19}$, die ihr Ideal in der kunstvollen (Hore) Vermählung (Windgötter) von Kreatürlichkeit und Geistigkeit hat.

19 Dass es sich bei der Geburt der Venus gleichsam um die Proklamation eines Ideals handelt, hat bereits Aby Warburg ( ${ }^{2} 1980$ [1979]: 39) angedeutet, als er sie gegen das andere Venusbild Botticellis, La Primavera, abhob, wo Venus bereits »in königlichem Schmuck in ihrem Reiche« erscheint. Für diese »idealistische« Deutung der Geburt der Venus spricht die überzeugende Interpretation der späteren Primavera, die Horst Bredekamp (1988: 83) vorgeschlagen hat. Seinem Nachweis zufolge ist dieses zweite Venus-Programm erkennbar von jenem irdischen Paradies abgerückt, das sich im frühen Bild noch programmatischer zu artikulieren scheint. 
Der Bezug auf Pico ist nicht beliebig. Er wie Poliziano und mit ihm Botticelli standen in enger Beziehung zu Marsilio Ficinos platonischer Akademie in Florenz. Manches spricht dafür, dass sie den philosophischen Hintergrund Botticellis bildet. In einem Brief an den Auftraggeber des Bildes erklärt er Venus als humanitas, Sinnbild sittlich vollkommenen Menschentums. ${ }^{20}$ Vor allem aber nennt er sie in Übereinstimmung mit Polizia$\mathrm{no}^{21}$ eine Nymphe! Nichts, was die Arkadier erleben, geht deshalb im vordergründigen Liebesstreit auf. Diese pastorale Gemeinde ist ihrerseits ein Projekt der Renaissance. Sannazaro, der Akademiker, wusste, was sich in seiner Kunstwelt vortragen ließ.

Poetisch sucht er seine Lösung in einem sermo humilis. ${ }^{22}$ Für ihn ist er das, was die Venus für Botticelli: sermo tritt für kulturelle Gestaltung, humilis für die natürlichen Antriebe ein. Wie aber ließe sich - dadurch - die liebende Entzweiung von Schäfer und Nymphe shuman « überwinden? Sannazaro hat dazu bereits in der ersten Abteilung der Arcadia ein faszinierendes Szenarium entworfen. Da ist Ergasto, ein Doppel des Autors, Inbegriff dessen, was arkadische Sprachkunst vermag; Modellschäfer für viele nach ihm. Wie es sich gehört, hat ihn, mit allen (melancholischen) Symptomen, die Liebeskrankheit erfasst. Das kam so. Eines schönen Tages fällt ihm die lichte Gestalt einer Nymphe ins Auge. ${ }^{23}$ Siè stand, entblößt, im Bach, singend - ein Bild dessen, was sie ausmacht: vermählt mit dem Wasser, aus dem das Leben kommt; ohne Verhüllung und Verstellung der Kultur, unverfälschter Ausdruck der Natur - eine Schwester der Venus. Vor allem: Sie singt, für sich, ohne Grund, nicht wie die gequälten Schäfer. Rousseau oder Schiller hätten sie für eine Übersetzung des `Naiven ‘ halten können.

Ein wahrhaft umstürzendes Geschehen setzt ein. Ihr natürlicher Zauber raubt ihm die Sinne: Ohnmächtig fällt er zu Boden. In Arkadien jedoch ist alles Zeichen. Durch sein jähes Begehren teilt sich dem Schäfer Ergasto auf natursprachlichem Wege mit, dass ihm etwas Grundlegendes fehlt. Draußen, in der Gesellschaft, würde es sich negativ, als Unbehagen in der Kul-

\footnotetext{
20 Wiedergegeben bei Jan Lauts (1958: 20-21).

21 Angelo Poliziano (1981: 113-114 [I, vv. 99-103]).

22 Ausdrückliches Stilprogramm im »Prologo«: "Onde io, se lecito mi fusse, più mi terrei a gloria di porre la mia bocca a la umile fistula di Coridone, $[\ldots]$, che a la sonora tibia di Pallade, [...] [Epos], in: Jacopo Sannazaro (1990: 55). Ebenso Torquato Tasso (1995: 12-13 [vv. 76ss.]).

23 Jacopo Sannazaro (1990: 62-63)
} 
tur anzeigen. Hier jedoch, im Anblick der Nymphe, wird es geradezu kairotisch identifiziert als eine Fülle, die seine Auffassung übersteigt. Danach ist Ergasto nicht mehr er selbst. Seine todesähnliche Geistesabwesenheit zeigt, dass er elementar von seiner alten Identität abgebracht wurde. Genau genommen ergeht es jedoch auch der Nymphe nicht anders. Sein fixierter Blick hat sie, die Unstete, Schweifende zutiefst befremdet: Jäh verstummt sie, bekleidet und verbirgt sich. Wollten sich so nicht Adam und Eva nach dem Sündenfall dem Blick des Herrn entziehen? Es ist Ausdruck auch für ihren Bruch des naiven Selbstverständnisses. Ihre Irritation wächst, als Ergasto, offensichtlich durch sie, wie tot zu Boden sinkt. Sie hatte den Erfolg der Jägerin, obwohl sie nicht gejagt hatte. Wohl konnte sie die natürlichen Zeichen des Todes lesen; unverständlich jedoch musste ihr die Ursache bleiben. Sie hatte sich eine Wirkung zuzuschreiben, von der sie bisher nichts wusste. Entsprechend reagiert sie. Angesichts des (scheinbar) leblosen Ergasto schlägt ihr Gesang deshalb in einen dissonanten Schrei um, Ausdruck dafür, dass sie sich am anderen selbst fremd geworden ist. Jetzt ist sie auf ihre Weise außer sich: Sie, die sonst Leben vernichtet, eilt ihm zu Hilfe. Und als Ergasto wieder zu sich kommt, flieht die, vor der sonst andere Wesen fliehen müssen. Auch ihre Ruh ist hin, wie später bei Fausts Gretchen.

Der Moment des innamoramento hat, anders als in der Welt der höfischen Liebe, die mitgebrachte Identität beider erschüttert. Die Blicke des Schäfers haben die Frau genauso getroffen, wie ihre Schönheit ihn. Einer hat den anderen jeweils von sich abgebracht und ihm eine tiefe Defizienzerfahrung zugefügt. Das Naturkind wird sich einer Macht bewusst, über die es selbst keine Macht hat. Das deckt zuletzt etwas Unbeherrschtes in ihrem naiven Wesen auf, ausgedrückt durch ihren - todbringenden - Jagdinstinkt. Der Kulturmensch dagegen wird, im höchsten Sinne des Wortes, bewusstlos. In der Begegnung des rationale mit dem animale geht jedem auf seine Weise auf, dass er im Grunde eine - unausgeglichene - Doppelnatur hat.

Was tut Ergasto, um sein anthropologisches Ungleichgewicht zu beheben? Was alle Schäfer tun: Er schweigt und sinnt (das petrarkistische »solo e pensoso $($ ), dann singt und klagt er und trägt seinen Kummer in die Rinde der Bäume ein. In Arkadien ist eben alles sprechend. Mit seinen Liedern und Zeichen holt er deshalb sprachlich ein, was die Nymphe ihm in der natürlichen Sprache der Sinne mitgeteilt hat. Von ihm aus gesehen handelt es sich um eine Rationalisierung ihrer Wirkung; denn er versucht, ihren über- 
wältigenden Zauber in Worte zu übersetzen und damit begreiflich zu machen. Seine Gesänge leisten damit Reflexionsarbeit: Er bildet seine innere Verletzung in der Sprache verletzter Liebe, im Klageton ab. Dabei wird ihm nach und nach bewusst, was sie ihm bedeutet.

Und sie? Sie meidet ihn. Doch Arkadien ist ein kleines Land mit hoher kommunikativer Durchdringung. Wenn sie ihm auch ausweicht, seine Lieder und Zeichen verfolgen sie überall hin. ${ }^{24}$ Von den sanften Anhöhen aus trägt der Ton weit hinaus; das Echo nimmt ihn mit; die Bäume halten es sichtbar fest; keinem bleibt es verborgen. Für die Nymphen war der Schäfer selbst nicht ansprechend; wohl aber haben sie einen Sinn für seinen Zauber: den Gesang und die Musik. Deswegen, heißt es ausdrücklich, legen sie sogar ihr Jagdwerkzeug nieder - ein bedeutsames Zeichen auch dies -, um den pastoralen Klängen zu lauschen. ${ }^{25}$ Und hier setzt die Peripetie im Liebesstreit und Selbstverständnis an. Indem sie auf die Wohllaute, die vertraute Natursprache hört, nimmt sie zugleich die Fremdworte auf, die er über sie sagt. Sie teilen ihr wieder und wieder mit, wer sie ist. So geht sie durch eine lange arkadische Sprachschule - die Schäfer müssen lange klagen, ehe sie erhört werden. Dadurch lernt die, die bisher unbewusst gelebt hat, sich nach und nach bewusst benennen. Aus ihrer Naturbegabung für das Melos entwickelt sich ein Sinn für den kulturellen Logos: Sie gewinnt einen Begriff von sich selbst.

Damit daraus aber ein Happy End werden kann, wie es nirgends anmutiger dargestellt wurde als in Tassos Aminta, muss sich jedoch auch der Schäfer einer anthropologischen Revision unterziehen. So sehr hat die Nymphe seine Sinne angesprochen, dass er seinen Verstand - rationale verloren hat. Würde sie sich ihm nicht entzogen haben, hätte seine Leidenschaft ins Unbeherrschte umschlagen können, wie beim Satyr, der mit Gewalt, also animalisch nimmt, was er braucht, wenn er kann. Deshalb muss Ergasto seinen jähen Liebeswunsch in einem langen Liebesdienst auf Dauer stellen, damit sein sinnlicher Aufruhr in ein erträgliches Verhältnis zu seinen geistigen Ansprüchen kommt. Das heißt aber nichts anderes, als dass sich seine bisherige Räson unter den Bedingungen ihrer kreatürlichen

24 Eine Art arkadische Primärkommunikation, Topos vieler Eklogen, zugleich Reminiszenz Petrarcas. Cf. beispielsweise einen Bezugspunkt auch Sannazaros, Angelo Poliziano, Fabula di Orfeo (1480), in: id. (1981: 140 [vv. 50ss.]): » [...] s'ella l'ode [scil. das Lied], verrà come una cucciola« (v. 91). Orpheus ist Schäfer und seine Sangesmacht Inbegriff pastoraler Logotherapie der Liebe.

Jacopo Sannazaro (1990: 54 [Prologo] sowie 65 [I]). 
Negation neu zu bilden hat. Darüber wird er selbst ein anderer. Ein hochsensibles Ereignis.

Die Schäfer haben, wenn sie von den Nymphen in Bann geschlagen werden, nichts anderes mehr im ১Kopf $\prec$ als sie. So unbedingt aber wie sie die Dienerinnen Dianas verfolgen, die von Verfolgung ja etwas verstehen, müssen diese sich dadurch nicht ganz als Objekt einer - tödlichen - Leidenschaft fühlen? ${ }^{26}$ Die Jägerinnen werden dadurch aus ihrer Sicht zu Gejagten. Das nötigt ihnen ihrerseits einen folgenreichen Rollenwechsel ab. Jetzt fliehen sie. Im Grunde aber ist es eine Flucht vor dem Bild, das die Schäfer sich von ihnen machen. Es mutet ihnen eine Selbsterfahrung zu, die ihnen nahe bringt, dass es ein heftiges Interesse an ihnen gibt, das sie nicht länger so sein lassen will, wie sie bisher waren. Ihr Widerstand ist im Grunde ihre Art von unbegrifflicher Reflexion. Er hält einerseits die Schäfer lange hin, sodass sie ihre Liebe - zunächst - als ihr eigenes, und in diesem Sinne ganz und gar subjektives Anliegen zur Kenntnis nehmen müssen. Die Nymphen zwingen sie also, diese subjektivistische Verengung zu objektivieren. Andererseits kann den Schäfern dabei aufgehen, dass die Naturverhaftung der Geliebten einem eigenen Konzept von Sittlichkeit und Sprache gehorcht. Allerdings müssen auch die rigiden Dienerinnen Dianas von ihrem objektivistischen Naturbann abgehen und ihn subjektivieren, indem sie von den Schäfern lernen, welchen Wert sie für sich selbst genommen haben. Jeder relativiert im Anderen seine eigene Einseitigkeit und wird dadurch offen für ein Projekt der Gegenseitigkeit - arkadische pietà. Sie anerkennt tätige, schöpferische Selbstvereinbarung des Menschen als höchste Tugend.

IV.

Von hier aus mag offenkundiger werden, warum Arkadien so viel zeitgenössische Aufmerksamkeit auf sich ziehen konnte. Das Land der Schäfer (und Nymphen) ist im höheren Sinne eine anthropologische Versuchsanstalt. Unter dem Schutz ihrer offensichtlichen Unwirklichkeit - jeder wuss-

26 Aus dem Orpheus-Mythos stammt das verbreitete Motiv, dass die Nymphe(!) Euridike auf der Flucht vor einem liebesrasenden Schäfer einen beispielhaft chthonischen Tod erleidet - sei es, dass sie im Wasser ertrinkt, sei es dass sie, in Anlehnung an den Sündenfall, von einer Schlange gebissen wird. Cf. dazu eine historisch einflussreiche Version bei Angelo Poliziano (1981: 145 [Fabula di Orfeo, vv. 190ss.]). 
te, dass sie eine Erfindung von humanistisch Gebildeten war - ließ sich der Anspruch freibleibender Selbstverwirklichung kühner erheben als in der überwachten Sprache zeitgenössischer Moralphilosophie. Denn die harmlos anmutenden Liebeshändel von Mann und Frau kehren im Grunde die theologisch-christlich zulässige Frage nach dem Menschen um. Bisher hatte er nach sich zu fragen: Was bin ich - im Blick auf ein absolutes Sein. Hier aber darf gelten: Was kann ich in Bezug auf mich selbst sein - abgesehen von meiner Einlassung in eine übergreifende Weltordnung, die sich mir entzieht. Das Bewusstsein des Menschen beginnt, sich auf eine differentielle Identität einzustellen.

Die Rollen von Schäfer und Nymphe sind dabei so verteilt, dass sie die Bedingungen dramatisieren, von denen eine solche Selbstbestimmung auszugehen hat. Sie anerkennen etwa in der Geschlechterdifferenz den Menschen als eine Doppelnatur, in der ein geistiges und ein sinnliches Prinzip ihr Recht fordern. Wie, so fragt es sich in Arkadien dringender als sonst wo, lässt sich dieser Streit beheben - ohne sich zuerst oder gar ausschließlich nach Tugendkatalogen zu richten, die mit der Autorität endgültiger, ewiger Lösungen auftreten. Darauf hat Sannazaro auf seine Weise eine erregende Antwort gegeben. Bemerkenswert daran ist, dass es ihm gerade nicht eigentlich um eine - gar verbindliche - Entscheidung ging. Das zeigt Sincero, ein anderer Schäfer und Doppel des Autors im Text. Er war mit gebrochenem Herzen aus Neapel nach Arkadien geflohen; und kehrt am Ende ohne sentimentale Heilung dahin zurück. Auch Ergasto, des Autors zweites alter ego, kommt nicht zur beseligenden Umarmung mit einer Nymphe, die Tasso seinen Figuren gewährt. Ihm lag weniger an einer Lösung als am Verfahren, wie sie herbeizuführen wäre.

Den Begriff des Menschen ihm selbst anheim zu stellen, verlangt im Grunde nach einer - anthropologischen - Kunst der Hermeneutik. In ihrem Sinne ist der Mensch Applikation seiner Wahlmöglichkeiten. ${ }^{27}$ Tasso andererseits wird, siebzig Jahre nach der Arcadia, bestätigen, dass selbst eine 'glückliche< Vereinbarung in Arkadien keinen Anspruch auf Dauer haben kann. Die beiden Altarkadier Dafne und Tirsi haben ihre erste Liebe bereits hinter sich und experimentieren mit ihren Zöglingen Silvia und Aminta an einem neuen Weg, der zum alten Ziel führt. Da das Was feststeht: das

27 Zur Thematisierung des Problems cf. Manfred Fuhrmann / Hans R. Jauß / Wolfgang Pannenberg (1981), wo der Applikation als festem Bestandteil der Hermeneutik zu ihrem Recht verholfen wird. 
Glück des Menschen, kommt es auf das Wie an. Dafür sorgt nicht zuletzt ein Figurant, der lange vor Poussins Bergers d'Arcadie Heimrecht in Arkadien hatte - »et in arcadia ego $\ll-$, der Tod. ${ }^{28}$ Wo seiner gedacht wird, etwa im fünften Kapitel der Arcadia, geschieht es weniger im Namen von Vergänglichkeit (und einem dazugehörigen carpe diem). Er steht vielmehr für die Übergänglichkeit des Lebens als solcher: Hinter dem Arkadien hier sind andere, schönere Arkadien denkbar. Glück ist, auch hierzulande, nicht vollkommen; es verdankt sich einer ständigen Anstrengung. Denn das ist die Kehrseite einer schöpferischen Freiheit zu sich selbst: Wer sie will, ruft zugleich mit ihr Proteus in die Welt, auch er eine Gottheit des Wassers wie die Abkömmlinge von Venus, die Nymphen. ${ }^{29}$ Er aber verkörpert gleichsam eine unerlöste Lebendigkeit, Metamorphose ohne Ziel, Freiheit der Selbstverwirklichung, die sich blind fortsetzt. Er verändert sich, statt die Rede über sich. Ihm fehlt die poetische Hermeneutik der Arkadier. Diese gedenken nicht zuletzt seiner, weil sie sich an ihm die neuen Schwierigkeiten vergegenwärtigen, die der Begriff eines Menschen mit sich bringt, der sich, innerhalb einer gegebenen Grammatik des Lebens - der natura naturata -, als offene Syntax - natura naturans - begreift. In Arkadien geht ein neues humanes Ideal an Land; dieses selbst ist, wie alle Ideale, unerreichbar schön, der Venus Botticellis vergleichbar. Seine Bewohner können deshalb nur ihr Streben, das heißt ihren Liebeswunsch, auf Dauer stellen. Glück ist, auch hier, nicht vollkommen; es verdankt sich einer unablässigen Anstrengung. Sie muss deshalb daran interessiert sein, weniger das Ziel als den Weg dahin, die Kunst, zu sichern. Die wahre Schönheit ihrer Humanität verkörpern daher ihre Lieder. An die Schwelle dieser Einsicht ist Sannazaro getreten. Allzu weit von der >Reformation< der Schriftauslegung ist dies nicht entfernt, die, vorbereitet von Nikolaus von Lyra, bei Melanchthon und Luther zur Erscheinung kommt, wo es heißt: „Sacra scriptura sui ipsius interpres. $\ll^{30}$

Die Arkadier sind dabei nicht ohne Leitbild vorgegangen; ganz auf sich bezogen zu sein, wird erst den Weltschmerz des modernen Subjekts ausma-

28 Cf. besonders das Prosimetrum V der Arcadia (1990: 95ss.) Zur Bildgenese bei Poussin cf. Milovan Stanic (1994).

29 Jacopo Sannazaro (1990: 112 [VI, vv. 51-54]): „[T]anto si può per arte il mondo involvere! / Èpico: Questo è Proteo, che di cipresso in elice, / e di serpente in tigre trasformavasi, / e feasi or bove, or capra, or fiume, or selice."

30 Cf. Winfried Wehle (1995: 153ss.) mit entsprechender Literatur. 
chen. Die paradiesische Naturfrömmigkeit des ersten Menschengeschlechts ist es, nach der sie ihre Lebensform ausrichten. Umso mehr, als sie in der vollendeten Form der Antike auf sie kam. Bestärkt durch deren ethische Autorität sollte jeder jederzeit bereit sein, dem Anderen zuzuhören, und durfte seinerseits hoffen, dass auch der Andere so für ihn da ist. Diese Rhetorik garantierte ihren Grundwert der pietà. Hinter ihr steht jedoch gleichwohl eine neuzeitliche These: dass der schöpferische Mensch in der Lage sei, seine Doppelnatur zu befrieden, nicht nur indem er sie - sich - überwindet, sondern sie vielmehr im Sinne des sanften Gesetzes dieses Landes vermittelt und dabei auf eine Gegenseitigkeit setzt, die in die Macht der Sprache gestellt ist. Noch Derridas Grammatologie steht im Banne dieser Logotherapie. Arkadien instituiert so gesehen die Sprache als ein Forum, wo der Mensch mit seinen Mitteln zu sich selbst kommen kann. Es krönt damit einerseits das humanistische Zutrauen in die Rhetorik, das sich auf Cicero berief. Andererseits lässt es in Umrissen bereits eine zukunftsweisende diskursive Identität erkennen. Als solche aber hebt sie sich grundlegend von herrschenden Tugendpraktiken ab. Dort kennt man die Natur des Menschen als Ursache seines Sündenfalls. Um die miseria hominis zu wenden, muss er deshalb der Ethik eines übernatürlichen Lebens unterzogen werden: Er kann nicht so bleiben, wie er ist. In dieser Absicht hat der Held der Epen das Böse, Zügellose, Heidnische in der Welt zu vernichten, um dem Gegenteil zur allein seligmachenden Anschauung zu verhelfen. Und der Christenmensch soll seine Selbstbezüglichkeit nutzen, um seine sündhaften Neigungen auszulöschen. So oder so ist ein entschiedener Mensch gefordert, der seine Kreatürlichkeit seiner Geistnatur aufopfert.

Arkadien aber will ihn gerade lassen, wie er ist. Hier gilt, dass, wer sprachlich aus sich herausgeht, den Anderen - die Andere - für sich einnimmt. Durch die Nymphe erfährt sich Ergasto auf schmerzliche Weise als seiner selbst entfremdet. Sie hat in seinem >kultivierten` Selbstverständnis ein Defizit an Vitalität aufgedeckt. Über seine Lieder aber kann er ihr nach und nach bewusst machen, dass umgekehrt ihrem Selbst eine höhere Bedeutung zukommt, als ihr sturer Naturgehorsam es zulässt. In Arkadien wird also dialogische Identität gelehrt. Mit ihr ist insofern schon ein Horizont von Subjektivität unterschieden, als der Mensch einen Begriff von sich wesentlich in Bezug auf den Menschen, nicht - nur - auf ein Übermenschliches zu bilden lernt. Dieser Übergang ist grundlegend. In dem Maße, wie er aus der Autorität einer theologischen oder kosmologischen Beziehung 
heraustritt, entdeckt er die dialogische Kommunikation als Weg einer tätigen Selbstbestimmung. Und er kann diesen Schritt wagen, weil dieses autonome Glücksvorhaben eine eigene Letztbegründung hinter sich weiß. Es ist die arkadische Idee, dass ursprünglich alles Geschaffene, also auch der Mensch, aufeinander >abgestimmt $<$ war und dieser Einklang in der wohltuenden Sprache von Musik und Gesang noch nachlebt. Die Panflöte, das Emblem der Hirten, soll für diese Neugeburt des Menschen aus dem Spielraum der Sprache bürgen.

V.

Natürlich, dieses selbstgemachte Glück kann nur >relativ`sein, nicht zu vergleichen mit einem himmlischen oder irdischen Paradies. Zeit, Befristung und Tod überschatten das schäferliche Bewusstsein. Mehr noch: Die Arkadier wissen und sagen es auch, dass sie das Glück der ersten Menschen nie wieder haben werden. Und ihr Publikum weiß, dass ihm selbst das relative Glück der Arkadier noch verwehrt bleiben wird. Deren Land ist eine Erfindung humanistischer Gelehrsamkeit ${ }^{31}$, eine >Fabel aus dem Reich des Menschenmöglichen, während sie selbst in der Realität einer >eisernen Zivilisation leben. Niemand konnte also ernsthaft glauben, durch imaginäre Sonntagsausflüge ins Land der Schäfer effektiv glücklicher zu werden. Aber zeitgenössische Sehnsüchte, allemal Defizite des historischen Alltags, nehmen dadurch diskursive Gestalt an: Sie werden so zu einer Redewirklichkeit. Gewiss lassen sich dadurch verlorene Paradiese nicht zurückbringen; aber sie bleiben so immerhin reflexiv, als Denkmöglichkeit am Leben. Darum vor allem ging es Sannazaro und seinen Nachahmern, auch wenn sie später mehr und mehr die Risiken dieser freien Selbstvereinbarung im Auge haben. ${ }^{32}$ Deshalb auch kam es ihm nicht zuerst auf die beseligende Umarmung von Hirte und Nymphe an, wie sie Tassos Aminta

31 Wie die Quellennachweise der Arcadia-Edition von Michele Scherillo (1888) belegen; cf. dazu ebenfalls Enrico Carrara (1952) in der Einleitung der Opere di Jacopo Sannazaro. Systematisch, als Stilprinzip untersucht von Maria Corti $\left({ }^{2} 1977\right.$ : 307-357). William J. Kennedy (1983: 115ss.) sieht in dieser »Dekonstruktion der Tradition« mit guten Gründen den Ansatz für eine neue polyphone Poetik, die ihre fiktive Identität am Ende (Kap. XI und XII) selbst wieder dekonstruiert.

32 So muss denn Arkadien als ein historisches Konzept neben anderen in der Entfaltung einer neuzeitlichen Anthropologie angesehen werden. Die Utopia des Thomas Morus wäre gleichsam als sein Gegenentwurf zu würdigen, der Cortegiano des Castiglione als eine auf gesellschaftliche Vernunft gegründete Variante. Cf. dazu Roland Galle (1990). 
feiern wird. Glück aus Menschenhand will überhaupt erst als Projekt gewonnen sein.

Der größte Beitrag Sannazaros dazu besteht aber wohl darin, dass er dieses Wunschbild der Poesie anvertraut hat. Der rettende Gedanke angesichts eines unwiederbringlichen Verlustes: Jeder hat, auch wenn er nicht mehr natürlich ist, noch immer Natur. Insofern müsste er, und sei es nur verkümmert, noch einen Reflex jener emphatischen Ursprache verspüren, welche die Kindheit des Menschengeschlechts so glücklich erscheinen lässt. Es käme also darauf an, im staub und stumm < gewordenen Menschen der Zivilisation an diese verschüttete Stimme der Natur zu appellieren. Eine solche diskursive Renaissance aber könnte nur noch der Kunst gelingen. Sie allein hat sich einen Sinn für die Sprache als Melos erhalten. Wer auf ihre >süßen< Klänge hört, versteht mehr, als der Wortlaut sagt. ${ }^{33}$ Um diese Erkenntnis ringt Sannazaro. Darin bestärkt ihn Giraldi Cinzio im Vorwort seines Satyrspiels Egle ${ }^{34}$, Tasso im Chorlied des zweiten Aktes seines Aminta. Sannazaro selbst weist voll Stolz am Ende darauf hin, dass er der erste nach Vergil gewesen sei, der die schlummernden Wälder wieder erweckt und den Schäfern die längst vergessenen Lieder singen gelehrt habe. ${ }^{35}$ Sein Aufenthalt dort diente also der Neubegründung der Dichtung hier. Arkadien ist, nach dem Rad des Vergil, der Anfang der Poesie. ${ }^{36}$ Zeichenhaft gehen deshalb die Nymphen und, in ihrem Gefolge, die Schäfer ad fontes. Sincero entdeckt auf ihrer Spur, als bizarre Traumallegorie inszeniert, eine Welt unterhalb Arkadiens, das heißt den Anfangsgrund aller Dichtkunst. Was sich oben als pastoraler Streit abspielt, ist nur der idyllische Widerschein eines Kampfes der großen Elemente, den Feuer und Wasser, Mars und Venus gegeneinander führen! ${ }^{37}$ Wenn Schäfer und Nymphe deshalb die menschliche Doppelnatur darstellen, so bilden sie im Grunde nur eine unhintergehbare mythische Polarität der Welt insgesamt ab. Als Sincero schließlich wieder an die Oberfläche gelangt, vermag er, als arka-

33 Unvergleichlich programmatisch von Tasso im Aminta als "nova eloquenza d'Amore« resümiert: "[S]pesso in un dir confuso / tu [scil. Amor] in bei facondi detti / sciogli la lingua dei fedeli tuoi e " $\mathrm{n}$ parole interrotte / meglio si esprime il core« (1995: 90ss.) - im Grunde eine Selbstcharakteristik des mragionar [...] in nuova guisa«, den er im Prolog (vv. 76-77) ankündigt.

34 Zwar habe die Natur ihre ursprünglichen Effekte verloren; aber der Dichter sei in der Lage, sie aus der »Höhle« der Vergangenheit zurückkommen zu lassen und damit ästhetisch die verlorene Natur zu restituieren. Cf. Giovan Battista Giraldi Cinzio (1980: $8 \mathrm{a} / \mathrm{b})$.

35 Jacopo Sannazaro (1990: 24-25).

36 Zum stilgeschichtlichen Zusammenhang cf. Werner Krauss (1976).

37 Jacopo Sannazaro (1990: 214ss. [XII]). 
disch Eingeweihter, ihre Zeichen überall zu lesen: im Gegensatz von Stadt und Land, im Verhältnis von Mann und Frau, im Unterschied von Berg und Tal, von künstlicher Fontäne und natürlicher Quelle.

Diese Einsicht in die Elementarnatur der Welt und des Menschen aber veranlasst ihn, seinen bisherigen Musen ein >Adieu zu sagen und die Ziergärten der akademischen Dichtkunst zu verlassen. Traditionsschweres Signal dafür ist, dass der Autor während der Rückkehr Sinceros dessen neapolitanische Minneherrin, das heißt seine bisherige akademische Dichtungsauffassung, den Tod der Euridike, der Beatrice und der Laura sterben lässt und ihm dadurch eine dichterische vita nuova abverlangt. Dieser neue Sang aber, dessen Notwendigkeit ihn Arkadien gelehrt hat, ist jedoch nicht die neue - arkadische Dichtung selbst, in der er sie sich erschließt! Sie wird erst von diesem Ursprung aus sichtbar. Das erfundene Land der Schäfer erweist sich auch in dieser Hinsicht als ein Ort der Reflexion: Nirgendwo lässt sich ursächlicher über Poesie nachdenken als in der Poesie. ${ }^{38}$ Was es dabei zu entdecken galt, sind die anthropologischen Quellen der Kunst. Sie liegen für Sannazaro und, repräsentativ für die Zeit der Renaissance, in der Antike, weil sie einen Sinn eben nicht für die Überwindung, sondern die Nachahmung der Natur des Menschen um ihrer selbst willen aufbringt.

Noch eine andere, nicht minder erhebliche Einsicht in das Wesen einer neuzeitlichen Kunst gewinnt Sincero in Arkadien. Gewiss kommt es den Schäfern auf das Liebesglück an. Im Vordergrund steht jedoch ein anderer Lohn ihres Gesanges: Es ist das Lob des Gesanges als solchem. Wo immer einer seine Stimme erhebt, einzeln oder im Wettstreit, kann er sicher sein, dass seine Kunst um ihrer selbst willen gewürdigt wird. Der eine wird dafür mit einem kostbaren Hirtenstab ausgezeichnet, der andere mit einer Flöte, einer Kithara, einem sprechenden Vogel und anderem mehr. Es ist Sannazaros metonymische Weise zu sagen, dass die Kunst einen Wert für sich selbst hat. Sie ist ein Drittes, hervorgegangen aus der Vermählung von Trieb und Geist, frei wie eine Metapher für immer neue Zusammenspiele. Nichts könnte ihre dignitas hominis besser veranschaulichen als die höchste Gabe, die Ergasto dem Sieger pastoraler Wettspiele verspricht: eine kunst-

38 Diese einer neuen Dichtkunst abverlangte Selbstreflexion wird in Arkadien selbst vollzogen, weil es dafür keine antiken poetologischen Vorgaben gibt, die sich kommentieren ließen. Dadurch wurde das Hirtenland, wie Wolfgang Iser (1991: 59) pointiert herausstellt, zu einem Ort, an »welchem literarische Fiktionalität durch das Kenntlichmachen ihres Fingiertseins in das Bewußtsein einer Epoche getrieben wurde $«$. 
voll bemalte Holzvase von Mantegna. Sie zeigt, inmitten eines üppigen bukolischen Dekors, eine nackte Nymphe. ${ }^{39}$ Kunst ist humaner Dienst an der Natur.

Das höchste Anliegen Arkadiens, so gibt Sannazaro zu verstehen, besteht demnach in der Reflexion auf das Anliegen Arkadiens. Es ist ein Land, wo man sich seiner Natürlichkeit noch bewusst werden kann. Wohl noch entscheidender aber ist, dass dieser anthropologische Anspruch ästhetisch erhoben wird: Die Schäfer singen; der Autor spricht poetisch über sie, weil sie poetisch den Autor vertreten. Wenn durch sie also ein neues Selbstverständnis des Menschen angeregt wird, dann ist es elementar an Kunst als seiner ermöglichenden Bedingung geknüpft. Nicht die Lebenswelt der Reformation und Gegenreformation, der Inquisition, des Scheiterhaufens, der fürstlichen Willkür, der Conquista, ist der Ort, wo der Mensch sich selbst bestimmen könnte. Einzig die Kunst gewährt ihm ein diskursives Neuland, in dem er in Gestalt von sprachlichen Entdeckungen unmittelbar zu sich selbst finden kann. In Arkadien geht daher auch eine Entdeckung in dem Sinne vor sich, dass hier mit poetischen Mitteln die Poesie als der kulturelle Spielraum schlechthin einsichtig wird, an dem das animal rationale die Entfremdung von Geist und Natur austragen kann. Da sie nach ihren Grenzen hin - Leib und Seele - bestimmt ist, kann sie nur dann »nach eigenem Ermessen « (Pico della Mirandola) frei sich selbst entfalten, wenn ihre Mitte selbst zwar nach außen eingegrenzt, nach innen aber unbestimmt ist. Diese Bedingungen einer freien Selbstermittlung konnte nicht nur damals - allein die Kunst erfüllen. Sie vermag die natursprachlichen Klänge, Zeichen und Gesten - die Nymphen - sich anzueignen und sie mit Hilfe des Kunstverstandes - die Schäfer - als eine verstandesferne Naturhaftigkeit kulturell zu fassen.

Der Umgang mit dem Naturhaften - könnte man nicht auch sagen: Eingeborenen? - wird dadurch nicht auf eine kämpferische, sondern verständige Grundlage gestellt. Auch hier gerät der Mensch durch ein befremdendes Anderes außer sich. Aber er setzt sich nicht darüber hinweg, sondern nimmt es, als das Andere seiner selbst, in seinen Begriff mit auf. Für die Entdeckung Amerikas kam diese Entdeckung Arkadiens allerdings zu spät.

39 Jacopo Sannazaro (1990: 200 [XI]). Cf. dazu Otto Kurz (1959). 


\section{Winfried Wehle}

\section{Bibliographie}

\section{Literarische Werke und andere Quellen}

Giraldi Cinzio, Giovan Battista (1980): Egle (1545), Nachdruck, Urbino: Quattro Venti. Pico della Mirandola, Giovanni (1990): Oratio de dignitate hominis, übers. v. N. Baumgartner, hrsg. u. eingel. v. August Buck, Hamburg: Meiner.

Poliziano, Angelo (1981): Stanze, Orfeo, Rime, hrsg. v. Sergio Marconi, Mailand: Feltrinelli.

Sannazaro, Jacopo (1888): Arcadia, hrsg. v. Michele Scherillo, Turin: Loescher.

Sannazaro, Jacopo (1990): Arcadia, hrsg. v. Francesco Erspamer, Mailand: Mursia.

Sannazaro, Jacopo (1952): Opere di Jacopo Sannazaro, hrsg. v. Enrico Carrara, Turin: UTET.

Tasso, Torquato (1995): Aminta, übers. u. hrsg. v. Janos Riesz, Stuttgart: Reclam.

\section{Forschungsliteratur}

Bredekamp, Horst (1988): Botticelli Primavera. Florenz als Garten der Venus. Frankfurt am Main: S. Fischer.

Burckhardt, Jacob (1952): Die Kultur der Renaissance in Italien. Ein Versuch, durchges. v. W. Goetz, Nachdruck der Urausgabe, Stuttgart: Kröner.

Cassirer, Ernst ( ${ }^{5} 1977$ [1927]): Individuum und Kosmos in der Philosophie der Renaissance, Darmstadt: Wissenschaftliche Buchgesellschaft.

Cody, Richard (1969): The Landscape of the Mind. Pastoralism and Platonic Theory in Tasso's "Aminta and Shakespeare's Early Comedies, Oxford: Clarendon.

Corti, Maria ( ${ }^{2} 1977$ [1969]): Metodi e fantasmi, Mailand: Feltrinelli.

Costa, Gustavo (1972): La leggenda dei secoli d'oro nella letteratura italiana, Bari: Laterza.

Curtius, Ernst R. (1948): Europäische Literatur und lateinisches Mittelalter, Bern: Francke.

Fuhrmann, Manfred / Jauß, Hans R. / Pannenberg, Wolfhart (Hrsg.) (1981): Text und Applikation. Theologie, Jurisprudenz und Literaturwissenschaft im hermeneutischen Gespräch, München: Fink.

Galle, Roland (1990): »Dialogform und Menschenbild in Castigliones Il Libro del Cortegiano«, Neohelicon 17, pp. 233-251.

Grimm, Reinhold R. (1981): »Allegorie«, in: Fuhrmann, Manfred / Jauß, Hans R. / Pannenberg, Wolfhart (Hrsg.): Text und Applikation. Theologie, Jurisprudenz und Literaturwissenschaft im hermeneutischen Gespräch, München: Fink, pp. 567-576.

Iser, Wolfgang (1991): Das Fiktive und das Imaginäre. Perspektiven literarischer Anthropologie, Frankfurt am Main: Suhrkamp.

Janik, Dieter (1994): »Die neuen Menschen der Neuen Welt. Zur gesellschaftlichen und kulturellen Rolle der mestizos«, in: id. (Hrsg.): Die langen Folgen der kurzen Conquista, Frankfurt am Main: Vervuert, pp. 49-74.

Janik, Dieter / Lustig, Wolf (Hrsg.) (1989): Die spanische Eroberung Amerikas. Akteure, Autoren, Texte. Eine Anthologie von Originalzeugnissen, Frankfurt am Main: Vervuert.

Kennedy, William J. (1983): Jacopo Sannazaro and the Uses of Pastoral, Hanover: University Press of New England. 
Krauss, Werner (1976): »Über die Stellung der Bukolik in der ästhetischen Theorie«, in: Garber, Klaus (Hrsg.): Europäische Bukolik und Georgik, Darmstadt: Wissenschaftliche Buchgesellschaft, pp. 140-164.

Kurz, Otto (1959): "Sannazaro and Mantegna« in: Studi in Onore di Riccardo Filangieri, 3 Bde., Neapel: L'Arte Tipografica, pp. 277-283.

Landmann, Michael (Hrsg.) (1962): „De homine«. Der Mensch im Spiegel seines Gedankens, Freiburg: Alber.

Lauts, Jan (1958): Sandro Botticelli. Die Geburt der Venus, Stuttgart: Reclam.

Levin, Harry (1969): The Myth of the Golden Age in the Renaissance, Bloomington / London: Indiana University Press.

Ordine, Nuccio (1999): Giordano Bruno und die Philosophie des Esels, München: Fink.

Stanic, Milovan (1994): Poussin, beauté de l'énigme, Paris: Place. (Revue d'esthétique)

Trabant, Jürgen (1997): »Sprache«, in: Wulf, Christoph (Hrsg.): Vom Menschen. Handbuch historische Anthropologie, Weinheim / Basel: Beltz, pp. $595 \mathrm{ff}$.

Warburg, Aby ( ${ }^{2} 1980$ [1979]): Ausgewählte Schriften und Würdigungen, hrsg. v. Dieter Wuttke, Baden-Baden: Koerner.

Wehle, Winfried (1995): »Columbus' hermeneutische Abenteuer«, in: id. (Hrsg): Das Columbus-Projekt. Die Entdeckung Amerikas aus dem Weltbild des Mittelalters, München: Fink, pp. 153-204.

Wehle, Winfried (1993): "Der Tod, das Leben und die Kunst. Boccaccios Decameron oder der Triumpf der Sprache«, in: Borst, Arno et al. (Hrsg.): Der Tod im Mittelalter, Konstanz: Universitäts-Verlag, pp. 221-260. 